rares et portent souvent sur des femelles croisées. L'Institut national de la Recherche agronomique a donc entrepris dès 1970, une comparaison des qualités d'élevage des femelles allaitantes de races Charolaise, Limousine et Maine-Anjou.

Un lot de génisses de race pure a été acheté dans chacune des races puis rassemblé dans un même troupeau en stabulation libre permanente pour être conduit selon un plan factoriel de croisement. Un lot de femelles Hereford maintenues en race pure, sert de référence. Les fécondations sont réalisées par inséminations artificielles durant 70 jours/an, les femelles de chaque race étant accouplées aux taureaux des trois races, une rotation des femelles accouplées à une même race de taureau est pratiquée chaque année. Pour limiter les difficultés du premier vêlage à 2 ans, un échantillonnage intra race des génisses et des taureaux a été effectué selon le même principe dans chacune des races.

A partir des performances relatives aux différents cycles de reproduction de ces femelles, nous avons comparé la valeur maternelle de ces trois races françaises pour les critères de productivité numérique et pondérale :

- malgré un poids au premier oestrus comparable, les Limousines ont une précocité sexuelle inférieure aux autres races. Cependant, leur taux de veaux sevrés annuellement par vache durant les trois années est supérieur à celui des Maine-Anjou qui ont elles aussi sevré plus de veaux que les Charolaises. Ces différences semblent liées, pour les Limousines, à une meilleure fertilité et, pour les Charolaises, à une plus grande mortalité des veaux;

- au-delà d'un an, les Charolaises et Maine-Anjou ont une croissance assez comparable ; leur poids sont toujours plus élevés que les Limousines et les Hereford. A quatre ans, les $M a i n e-A n-$ jou sont plus hautes au garrot que les Charolaises et Limousines. Par contre les Charolaises et Maine$A n j o u$ ont une longueur corporelle assez voisine mais supérieure aux Limousines. Leur ouverture pelvienne rapportée au poids de naissance de leurs veaux, explique la meilleure aptitude au vêlage des femelles Limousines comparées aux autres femelles. Enfin, les différences entre races maternelles de poids des veaux au sevrage sont étroitement associées aux différences, d'une part, de production laitière des mères et, d'autre part, de potentiel de croissance que celles-ci transmettent à leurs veaux.

Bien qu'il soit vain de conclure dès maintenant sur la valeur maternelle relative de ces races, nous avons estimé le poids de veaux sevrés annuellement par vache entretenue ramenée à son poids métabolique. Ce premier bilan ne tient pas compte de la valeur de réforme des mères et de la vraie valeur d'engraissement des veaux sevrés. Ainsi la recherche de systèmes d'exploitation plus élaborés pour ces races maternelles et utilisant au mieux la complémentarité entre leurs aptitudes respectives, reste un des principaux objectifs de notre expérimentation.

\title{
APTITUDES MATERNELLES DES RACES LAITIÈRES, MIXTES, RUSTIQUES ET A VIANDE, POUR LA PRODUCTION DE VEAUX DE BOUCHERIE EN FERME PAR CROISEMENT INDUSTRIEI
}

J. L. Foulley, F. Ménissier, J. Gaillard*. - Département de Génétique animale, C. N. R. Z. 78350 Jouy en Josas (France). * Institut Technique de l'Élevage bovin, 149, $R$. de Bercy, 75012 Paris (France).

Les composantes maternelles de diverses races laitières, rustiques et à viande utilisées en croisement industriel ont été analysées et comparées entre elles à partir du contrôle de descendance en ferme de taureaux Limousins, Blonds d'Aquitaine et Charolais comportant respectivement 374,94 et 256 descendances paternelles de 16765,4696 et 12824 veaux purs et croisés.

Les caractères étudiés concernent la note et les fréquences des difficultés de vêlage des femelles, le poids à la naissance, le poids à 75 jours, le pointage de conformation des veaux et le produit de ces deux dernières variables. Les effets additifs des différentes races de mères (I2, ro et I 3 respectivement croisées aux races paternelles Limousine, Blonde d'Aquitaine et Charolaise) ont été estimées, séparément pour chaque race de pères, par la méthode des moindres carrés avec correction des effets du sexe et du rang de vêlage et exprimées en écarts à l'estimée de la Frisonne.

Sur la base des notes et fréquence des difficultés de vêlage, les races maternelles se classent dans l'ordre décroissant d'aptitude dans les groupes suivants $=\mathrm{r}$ Aubvac, Taventaise; 2 Salers, Gasconne, Abondance, Montbéliarde ; 3 Charolaise, Normande; 4 Limousine, Brune des Alpes; 5 Frisonne, Blonde d'Aquitaine, 6 Pie Rouge de l'Est. La classification sur le poids à 75 jours et la croissance postnatale fait ressortir une supériorité d'ensemble des races laitières par rapport aux races à viande et rustiques d'autant plus nette que le potentiel de croissance de la race paternelle est élevé. Le pointage de conformation bouchère différencie sans ambiguïté les groupes de races à viande, mixtes et laitières, rustiques dans cet ordre d'appréciation décroissante. Sur la valeur syn- 
thétique bouchère, si ce dernier classement demeure inchangé, en croisement avec des mâles Limousins et Blonds d'Aquitaine, en Charolais toutefois les races laitières de grand format comme la Pie-Rouge de l'Est, la Brune des Alpes et la Normande permettent une meilleure expression du potentiel de croissance de ces mâles.

Ces résultats indiquent notamment que la valorisation des races comme femelles allaitantes support d'un croisement avec des races à viande, implique la recherche d'une complémentarité entre les composantes maternelles des premières et directes des secondes, en vue d'obtenir un équilibre optimum entre le format, les caractères de croissance (potentiel de croissance, production laitière) et la conformation.

\title{
APTITUde A LA GÉMELLITÉ DES RACES A VIANDE FRANÇAISES : OBSERVATIONS EN ÉLEVAGE ET CONSTITUTION D'UN TROUPEAU DE SÉLECTION
}

\author{
F. Ménissier, J. Frebling. - Département de Génétique animale, C. N. R. Z., 78350, Jouy en \\ Josas (France).
}

Du fait des récents progrès dans les techniques de reproduction et de l'existence d'une variabilité d'origine génétique de la fréquence des vêlages gémellaires, il est envisageable d'accroître par sélection le taux de gémellité chez les bovins de races à viande.

A partir des informations collectées en ferme lors du contrôle national decroissance des bovins, nous avons estimé la fréquence des naissances gémellaires dans les races à viande françaises en fonction du rang de vêlage des mères et de la saison : cette fréquence est environ 7 fois plus élevée en Maine-Anjou (3,8 et 5,3 p. 100) qu'en Limousine (0,5-0,8 p. 100) ; la Charolaise $(3,2-3,4$ p. 100) et la Blonde d'A quitaine (1, I-0,9 p. roo) ont des fréquences intermédiaires. Ces résultats confirment nos précédentes observations où nous avions noté des différences entre régions et une certaine répétabilité entre années du taux de veaux jumeaux des grands élevages. A cette occasion, nous avions aussi constaté que, si la fréquence des couples de jumeaux monozygotes parmi l'ensemble des couples de jumeaux diminue avec l'âge des mères, leur fréquence absolue rapportée à l'ensemble des vêlages reste faible $(0,4 \mathrm{p}$. roo) et peu variable avec le rang de mise bas des mères.

Dès I97I-1972 nous avons rassemblé dans un même troupeau des vaches ayant eu naturellement 2 à 5 fois des veaux jumeaux. Ces $2 x$ Maine-Anjou et I 8 Charolaises constituent le troupeau de femelles fondatrices que nous avons exploité durant 3 années en race pure, 48 et 41 p. Ioo respectivement de leurs mises bas dans leurs élevages d'origine, avaient donné naissances à des veaux jumeaux.

Après ces 3 années, 19 couples de jumeaux sont nés (32 p. 100 des vêlages). A la suite des premiers vêlages observés dans le troupeau, mais résultant des fécondations dans les élevages d'origine des vaches fondatrices, 44 p. Ioo des Maine-Anjou et I2 p. Ioo des Charolaises ont engendré des veaux jumeaux. En ne considérant que les fécondations obtenues dans le troupeau, 24 et 36 p. roo des vêlages de ces races respectivement ont produit des veaux jumeaux. Parmi les 28 premières fondatrices exploitées durant deux campagnes dans les mêmes conditions, 7 (25 p. I0o) ont à nouveau donné au moins une fois des jumeaux. Si nous rassemblons toutes les performances des vaches fondatrices, il ressort alors que 23 p. Ioo d'entre elles ont eu naturellement au moins 4 paires de jumeaux.

Ces premiers résultats sont encourageants. La poursuite de ce travail nécessite, d'une part la recherche de critères précoces prédicteurs de l'aptitude à la gémellité et, d'autre part, le recours aux techniques de superovulation et transplantation d'œufs. C'est dans de telles conditions que nous allons tenter de préciser les modalités d'application d'une sélection efficace sur l'aptitude à la gémellité.

\section{WIRTSCHAFLICHE AUSWIRKUNGEN DER EINKREUZUNG} VON HOLSTEIN-FRIESTANS IN DEUTSCHE SCHWARZBUNTE RINDER

\section{E. ERnst. - Institut für Tierucht und Tierzhaltung, Universität Kiel (BRD).}

Die Einkreuzung von Holstein-Friesians in deutsche schwarzbunte Rinder hat die Ausgangspopulation der deutschen Rinder verändert. $F_{1}$-Tiere und Deutsche Schwarzbunte unterscheiden sich in wichtigen Merkmalen signifikant. $\mathrm{F}_{1}$-Tiere übertreffen die deutschen Schwarzbunten im Gewicht um 6 Prozent in der Widerristhöhe um 4 Prozent in der Milchleistung um ${ }_{3} 3$ Prozent 